\title{
Soft Independent Modeling of Class Analogy for Classifying Lumber Species Using Their Near-infrared Spectra ${ }^{1}$
}

\author{
Sang-Yun Yang ${ }^{2}$ Yonggun Park ${ }^{2,3} \cdot$ Hyunwoo Chung ${ }^{2} \cdot$ Hyunbin Kim $^{2} \cdot$ Se-Yeong Park ${ }^{2,3}$ • \\ In-Gyu Choi ${ }^{2,3,4} \cdot$ Ohkyung Kwon ${ }^{5} \cdot$ Hwanmyeong Yeo ${ }^{2,3, \dagger}$
}

\begin{abstract}
This paper examines the classification of five coniferous species, including larch (Larix kaempferi), red pine (Pinus densiflora), Korean pine (Pinus koraiensis), cedar (Cryptomeria japonica), and cypress (Chamaecyparis obtusa), using near-infrared (NIR) spectra. Fifty lumber samples were collected for each species. After air-drying the lumber, the NIR spectra (wavelength $=780-2500 \mathrm{~nm}$ ) were acquired on the wide face of the lumber samples. Soft independent modeling of class analogy (SIMCA) was performed to classify the five species using their NIR spectra. Three types of spectra (raw, standard normal variated, and Savitzky-Golay $2^{\text {nd }}$ derivative) were used to compare the classification reliability of the SIMCA models. The SIMCA model based on Savitzky-Golay $2^{\text {nd }}$ derivatives preprocessing was determined as the best classification model in this study. The accuracy, minimum precision, and minimum recall of the best model (PCA models using Savitzky-Golay $2^{\text {nd }}$ derivative preprocessed spectra) were evaluated as $73.00 \%$, 98.54\% (Korean pine), and 67.50\% (Korean pine), respectively.
\end{abstract}

Keywords: near-infrared spectroscopy, soft independent modeling of class analogy, non-destructive species classification, lumber classification, domestic species

\section{INTRODUCTION}

Lumber is used in various applications, such as structure, furniture, and packaging that require good physical properties. The species is an important factor in lumber's performance. Thus, it is traded at different prices for each species. Lumber species identification is an important process for ensuring market reliability. However, traditional species identification methods, such as anatomical analysis or DNA analysis, are not easily applicable. Both approaches, which are promising in lumber species identification, inevitably require cutting and processing specimens into a suitable form for examination. The test procedures take a long time

${ }^{1}$ Date Received December 21, 2018, Date Accepted January 14, 2019

2 Department of Forest Sciences, Seoul National University, 1 Gwanak-ro, Gwanak-gu, Seoul, 08826, Republic of Korea

${ }^{3}$ Research Institute of Agriculture and Life Sciences, Seoul National University, 1 Gwanak-ro, Gwanak-gu, Seoul, 08826, Republic of Korea

${ }^{4}$ Institutes of Green Bio Science and Technology, Seoul National University, 1447 Pyeongchang-daero, Daehwa-myeon, Pyeongchang, 25354, Republic of Korea

${ }^{5}$ National Instrumentation Center for Environmental Management, Seoul National University, 1 Gwanak-ro, Gwanak-gu, Seoul, 08826, Republic of Korea

† Corresponding author: Hwanmyeong Yeo (e-mail: hyeo@snu.ac.kr, ORCID:0000-0002-1779-069X) 
Sang-Yun Yang $\cdot$ Yonggun Park $\cdot$ Hyunwoo Chung $\cdot$ Hyunbin Kim $\cdot$ Se-Yeong Park

In-Gyu Choi $\cdot$ Ohkyung Kwon $\cdot$ Hwanmyeong Yeo

and cost. In addition, skilled experts are essential to analyze lumber species but they are a few in Korea. In field, visual species identification is preferred but it is based on subjective judgments of inspector and is applicable only limited species. To overcome the above-mentioned problems, alternative species identification techniques, such as machine vision (Hermanson and Wiedenhoeft, 2011; Hafemann et al., 2014; Kwon et al., 2017) and near-infrared (NIR) spectroscopy (Adedipe et al., 2008; Russ et al., 2009; Nisgoski et al., 2017; Park et al., 2017), have been reported. This study applies simple and non-destructive species classification based on NIR spectroscopy.

NIR spectroscopy is a type of analytical method that characterizes the chemical composition of a material. NIR rays are electromagnetic waves of wavelength range from $780 \mathrm{~nm}$ to $2,500 \mathrm{~nm}$ that have a sufficient energy level to vibrate molecular functional groups. The advantages of NIR spectroscopy are its nondestructive testing and rapid measurement capabilities (Blanco, 2002; Pasquini, 2003; Porep et al., 2015). In wood science, NIR application has been widely studied to determine the wood's physical properties (Schimleck and Evans, 2003; Jiang et al., 2006; Yang et al., 2017), chemical aspects (Alves et al., 2006; Watanabe et al., 2006; Üner et al., 2011; Cho et al., 2016), mechanical strength (Thumm and Meder, 2001; Zhao et al., 2009; Hovarth et al., 2011), and moisture content (Thygesen and Lundqvist, 2000; Eom et al., 2010; Chang et al., 2015; Yang et al., 2015). In this context, some classification studies using NIR spectroscopy have recently been reported because chemical composition varies by species. However, there are few reports of the lumber classification among domestic species (Hwang et al., 2015).

This study examines NIR spectroscopy with soft independent modeling of class analogy (SIMCA) to classify five domestic lumber species. SIMCA (Wold, 1976) is a statistical classification method for supervised pattern recognition and is widely applied in fields, such as chemometrics. NIR spectra acquired from lumbers were used for SIMCA modeling, and the classification reliability of each model was then evaluated.

\section{MATERIALS and METHODS}

\subsection{Sample preparation}

Fifty green lumbers of each species [larch (Larix kaempferi), red pine (Pinus densiflora), Korean pine (Pinus koraiensis), cedar (Cryptomeria japonica), and cypress (Chamaecyparis obtusa)], of dimensions $50 \times$ $100 \times 600 \mathrm{~mm}$ (thickness $\times$ width $\times$ length, $\mathrm{R}$ or $\mathrm{T}$ $\times \mathrm{L}$ direction), were collected from several National Forestry Cooperative Federations located throughout Korea (Table 1). These five species accounted for the majority of the log supplied to the domestic lumber production industry. Each sample was air-dried at $25^{\circ} \mathrm{C}$ and $65 \pm 10 \%$ relative humidity for 3 months. After air drying (10-15\% moisture content), lumbers were planed (2 mm thickness) for NIR measurement.

Table 1. The number of lumber samples collected from several National Forestry Cooperative Federations

\begin{tabular}{c|c|c|c|c|c}
\hline Species & Larch & $\begin{array}{c}\text { Red } \\
\text { pine }\end{array}$ & $\begin{array}{c}\text { Korean } \\
\text { pine }\end{array}$ & Cedar & Cypress \\
\hline \hline Yeoju & 10 & 0 & 0 & 0 & 0 \\
\hline Gapyeong & 10 & 20 & 50 & 0 & 0 \\
\hline Donghae & 10 & 10 & 0 & 0 & 0 \\
\hline Naju & 10 & 20 & 0 & 10 & 10 \\
\hline Namwon & 10 & 0 & 0 & 10 & 20 \\
\hline Seogwipo & 0 & 0 & 0 & 30 & 20 \\
\hline
\end{tabular}

\subsection{Acquisition of near-infrared (NIR) spectra}

All NIR absorbance spectra were acquired using a SpectraStar 2600 XT-R spectrometer (Unity Scientific, US). The NIR absorbance spectra were collected at 1 
Soft Independent Modeling of Class Analogy for Classifying Lumber Species Using their Near-infrared Spectra

$\mathrm{nm}$ intervals over the wavelength range 780 to 2,500 $\mathrm{nm}$ from the heartwood on the lumber's widest face. In total, twelve scans were averaged into a single spectrum. The lumbers were placed on the NIR acquisition window $(25 \times 40 \mathrm{~mm})$ of the spectrometer and then five spectra were obtained at different points on the same face. After NIR acquisition, a $2 \mathrm{~mm}$ thickness was planed, and this process was repeated four times. Thus, the NIR spectra were acquired from 20 different points for each lumber. As a result, 1,000 spectra were acquired for each species.

\subsection{Soft independent modeling of class analogy (SIMCA)}

The SIMCA classification model was developed using The Unscrambler 10.3 (CAMO, Norway) software. The NIR spectral data of each species were randomly divided into a training set (800 spectra) and test set (200 spectra). In SIMCA, a class (species in this study) can be modeled by means of principal components analysis (PCA). As a separate PCA is performed for each training set, information of dataset is extracted by orthogonalizing the variance of data and storing this in the principal components (PCs). The optimal number of PCs for the PCA model was determined when the increment of the total explained variance with added PC was $<1 \%$, to prevent overfitting (Yang et al., 2013).

In the SIMCA classification procedure, every spectrum of the training set was subjected to each optimal PCA model. Then, the residuals of each classspecific PCA model were evaluated to define the distribution of residuals for each class training set, thereby allowing classification of a new sample to one or several available classes (Bylesjö et al., 2006; Fujimoto and Tsuchikawa, 2010). Given this class-specific residual distribution, any spectra in the test set can subsequently be classified with a probability of equal variance compared to the model residuals according to Fisher's test ( $\mathrm{F}$-test). Outliers were detected in the $75 \%$ confidence interval of the $\mathrm{F}$-distribution in our study. The results of the F-test of residuals by SIMCA show that there are three possible results of classification (Esbensen et al., 2002); (1) a sample belongs to a class, (2) a sample belongs to several classes, or (3) a sample does not belong to a class. In this study, we defined the second result as multi-classified and the third as unclassified. Classification reliability was evaluated using the test set. For SIMCA modeling, different types of NIR data were used: original spectra, standard normal variate (SNV) preprocessed spectra, and SavitzkyGolay $2^{\text {nd }}$ derivative (window size $=21$, polynomial order $=2$ ) preprocessed spectra. This approach was taken because model performance based on NIR spectra could differ by mathematical preprocessing.

\section{RESULTS and DISCUSSION}

\subsection{Near-infrared (NIR) spectra}

Fig. 1 shows the raw NIR spectra of each species (the spectra for each species training set were averaged to give a single spectrum). The raw spectra had a different absorbance, depending on the species. NIR light penetrates less than visible light, so thick material, such as lumber, acquires an absorbance spectrum in a diffuse reflectance mode. Even if they are the same species, the absorbance differs, depending on the surface roughness and grain angle in the lumber. These factors make the spectral variance more than a species difference. Therefore, it is necessary to preprocess the NIR spectra, to ensure the analysis' reproducibility. In this study, SNV (Fig. 2) and the Savitzky-Golay $2^{\text {nd }}$ derivative (Fig. 3) preprocessing were applied to the spectra. The results of SNV preprocessing (Barnes et al., 1989) showed that each species' average spectrum was more similar than the raw absorbance spectra. There 
Sang-Yun Yang $\cdot$ Yonggun Park $\cdot$ Hyunwoo Chung $\cdot$ Hyunbin Kim $\cdot$ Se-Yeong Park

In-Gyu Choi $\cdot$ Ohkyung Kwon $\cdot$ Hwanmyeong Yeo

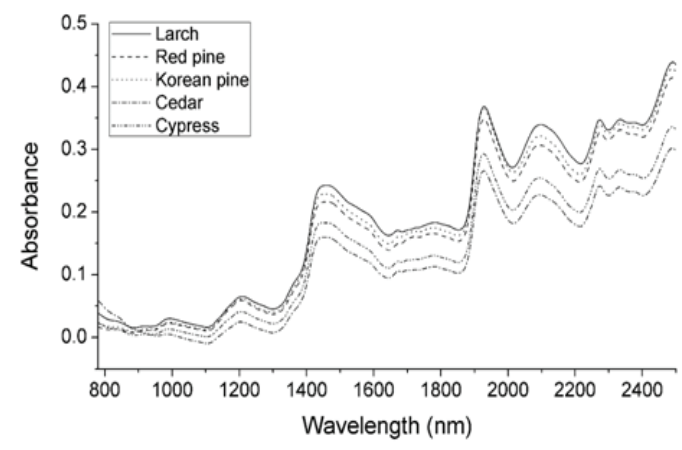

Fig. 1. Raw average NIR absorbance spectra for each species.

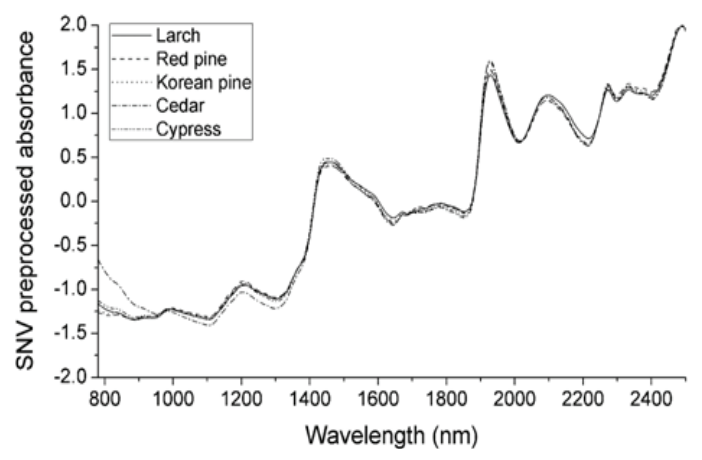

Fig. 2. SNV preprocessed average NIR absorbance spectra for each species.

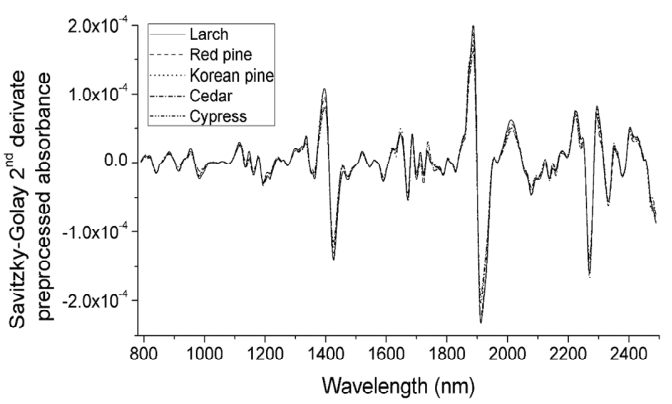

Fig. 3. Savitzky-Golay $2^{\text {nd }}$ derivative preprocessed average NIR spectra for each species.

was a difference in the absorbance of cedar in the below $1,100 \mathrm{~nm}$ region compared to other species. The Savitzky-Golay $2^{\text {nd }}$ derivative preprocessing can deconvolute the overlapping of absorption bands and remove the baseline (Savitzky and Golay, 1964). Fig. 3 shows the Savitzky-Golay $2^{\text {nd }}$ derivative preprocessed average absorbance spectra. It was absolute that the spectral pattern for each species' average spectra was also more similar than the raw absorbance spectra. Thus, it was expected that the variance originated from species variation would be more dominant after mathematical preprocessing.

\subsection{Principal components analysis (PCA) modeling}

Table 2 shows the optimal number of PCs, and the total explained variance in the optimal PCs for each class. PCA models of each species, using the raw spectra, had optimal PCs when one or two PCs were included. The total explained variance of the optimal model was about $99 \%$, which meant that only one or two PCs contained $99 \%$ of the raw spectra information. This outcome is because the absorbance highly correlates with the overlapping of the absorption bands, and the baselines were not removed. The PCA models for each species, using the SNV preprocessed spectra, were evaluated as optimal when they had 5-7 PCs that explained about $96-98 \%$ of the total variance. The SNV preprocessed PCA models had more PCs but fewer variances than the raw PCA models. These results were also found in the PCA models based on the SavitzkyGolay $2^{\text {nd }}$ derivative preprocessed spectra, which had 6-9 optimal PCs and explained $89-91 \%$ of the total variance. This means that mathematical preprocessing weakened correlation of the raw NIR data and emphasized the characteristics of the data.

\subsection{Soft independent modeling of class analogy (SIMCA) classification}

The performance of a classification model can be evaluated by calculating the number of correctly 
Soft Independent Modeling of Class Analogy for Classifying Lumber Species Using their Near-infrared Spectra

Table 2. Optimal number of principal components and explained total variance of principal component analysis model

\begin{tabular}{|c|c|c|c|c|c|c|c|c|c|c|}
\hline \multirow{2}{*}{$\begin{array}{l}\text { NIR data } \\
\text { type }\end{array}$} & \multicolumn{2}{|c|}{ Larch } & \multicolumn{2}{|c|}{ Red pine } & \multicolumn{2}{|c|}{ Korean pine } & \multicolumn{2}{|c|}{ Cedar } & \multicolumn{2}{|c|}{ Cypress } \\
\hline & $\mathrm{PCs}^{*}$ & $s_{t o t}{ }^{* *}$ & PCs & $s_{t o t}$ & PCs & $s_{t o t}$ & PCs & $s_{\text {tot }}$ & PCs & $s_{\text {tot }}$ \\
\hline Raw & 2 & $99.33 \%$ & 2 & $99.10 \%$ & 2 & $99.28 \%$ & 2 & $98.71 \%$ & 1 & $98.98 \%$ \\
\hline $\mathrm{SNV}^{* * *}$ & 7 & $97.55 \%$ & 7 & $96.73 \%$ & 6 & $96.05 \%$ & 5 & $97.00 \%$ & 6 & $98.32 \%$ \\
\hline SG $2 \mathrm{nd}^{* * * *}$ & 7 & $91.30 \%$ & 9 & $91.11 \%$ & 9 & $91.93 \%$ & 8 & $89.54 \%$ & 6 & $91.11 \%$ \\
\hline $\begin{array}{ll}* & \text { optimal } \\
* * & \text { explaine } \\
* * * & \text { Standarc } \\
* * * * & \text { Savitzky }\end{array}$ & $\begin{array}{l}\text { mber o } \\
\text { otal va } \\
\text { ormal } \\
\text { Golay }\end{array}$ & $\begin{array}{l}\text { rincipal c } \\
\text { ace contai } \\
\text { ate } \\
\text { derivative }\end{array}$ & nen & numbe & . & omn & & & & \\
\hline
\end{tabular}

Table 3. Confusion matrix in the case of binary classification

\begin{tabular}{c|c|c}
\hline Actual class & Positive & Negative \\
\hline \hline Predicted class & $\begin{array}{c}\text { True positive } \\
\text { (TP) }\end{array}$ & $\begin{array}{c}\text { False positive } \\
\text { (FP) }\end{array}$ \\
\hline Negative & $\begin{array}{c}\text { False negative } \\
\text { (FN) }\end{array}$ & $\begin{array}{c}\text { True negative } \\
\text { (TN) }\end{array}$ \\
\hline
\end{tabular}

classified class samples (true positives, TP), the number of correctly classified samples that do not belong to the class (true negatives, TN), and the samples that were either incorrectly classified (false positives, FP) or were not classified as class samples (false negatives, FN). These four counts constitute a confusion matrix, as shown in Table 3, in the case of the binary classification (Sokolova and Lapalme, 2009).

Accuracy is defined as the number of correctly classified positive and negative samples divided by the total sample number (Eq. 1). Precision is the number of correctly classified positive samples divided by the number of samples predicted as positive (Eq. 2). Recall is the number of correctly classified positive samples (TP) divided by the number of actual positive samples (Eq. 3).

$$
\begin{array}{r}
\text { Accuracy }(\%)=\frac{T P+T N}{T P+F P+F N+T N} \times 100 \cdots \\
\text { Precision }(\%)=\frac{T P}{T P+F P} \times 100 \cdots \cdots \cdots . . . \\
\text { Recall }(\%)=\frac{T P}{T P+F N} \times 100 \cdots \cdots \cdots \cdots
\end{array}
$$

Table 4 presents the species classification result (confusion matrix) of SIMCA based on raw spectra, for which the classification accuracy was $35.50 \%$. The precisions for each species were in the range 78.9599.07\%. However, the recalls for each species were very low, at $15.00-57.50 \%$. An interesting point is that the total number of misclassified samples was much fewer than that of multi-classified or unclassified samples. Among the multi-classified samples, there were two to even five class multi-classifications (not shown in this study). As the reliability parameters were evaluated as very low, it was difficult to expect clear species classification using SIMCA with raw spectra.

Table 5 provides the species classification result of SIMCA based on SNV preprocessed spectra, for which the classification accuracy was $51.90 \%$. The precisions for each species were in the range 90.67-100.00\%. However, recalls for each species were very low, in the range $19.50-76.50 \%$. Accuracy, precision, and recall were improved after SNV preprocessing of the raw spectra, except for the precision for cedar and recall for cypress. The number of unclassified samples was similar to that of the SIMCA model based on raw spectra. However, the number of multi-classified samples was highly reduced, except for cypress. In the case of cypress, the almost multi-classified samples were assigned as both cedar and cypress (not shown in this paper). It was estimated that the spectral pattern of 
Sang-Yun Yang $\cdot$ Yonggun Park $\cdot$ Hyunwoo Chung $\cdot$ Hyunbin Kim $\cdot$ Se-Yeong Park $•$ In-Gyu Choi $\cdot$ Ohkyung Kwon $\cdot$ Hwanmyeong Yeo

Table 4. Confusion matrix of SIMCA based on each species PCA models using raw spectra

\begin{tabular}{c|c|c|c|c|c|c}
\hline Actual species & Larch & Red pine & Korean pine & Cedar & Cypress & Precision (\%) \\
\hline \hline Predicted species & 106 & 0 & 1 & 0 & 0 & 99.07 \\
\hline Larch & 0 & 30 & 6 & 0 & 2 & 78.95 \\
\hline Red pine & 1 & 3 & 32 & 0 & 1 & 86.49 \\
\hline Korean pine & 1 & 1 & 0 & 115 & 2 & 96.64 \\
\hline Cedar & 2 & 1 & 2 & 5 & 72 & 87.80 \\
\hline Cypress & 52 & 48 & 36 & 60 & 29 & - \\
\hline Unclassified & 38 & 117 & 123 & 20 & 94 & - \\
\hline \hline Multi-classified & 200 & 200 & 200 & 200 & 200 & - \\
\hline Total (ea) & 53.00 & 15.00 & 16.00 & 57.50 & 36.00 & - \\
\hline Recall (\%) & \multicolumn{2}{l}{35.50} & & \\
\hline
\end{tabular}

Table 5. Confusion matrix of SIMCA based on each species PCA models using Standard normal variate preprocessed spectra.

\begin{tabular}{|c|c|c|c|c|c|c|}
\hline $\begin{array}{l}\text { Actual species } \\
\text { Predicted species }\end{array}$ & Larch & Red pine & Korean pine & Cedar & Cypress & Precision (\%) \\
\hline Larch & 153 & 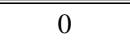 & 0 & 0 & 0 & 100.00 \\
\hline Red pine & 0 & 94 & 3 & 0 & 0 & 96.91 \\
\hline Korean pine & 0 & 5 & 97 & 0 & 0 & 95.10 \\
\hline Cedar & 0 & 1 & 0 & 136 & 13 & 90.67 \\
\hline Cypress & 0 & 0 & 0 & 0 & 39 & 100.00 \\
\hline Unclassified & 43 & 56 & 43 & 64 & 27 & - \\
\hline Multi-classified & 4 & 44 & 57 & 0 & 121 & - \\
\hline Total (ea) & 200 & 200 & 200 & 200 & 200 & - \\
\hline Recall (\%) & 76.50 & 47.00 & 48.50 & 68.00 & 19.50 & 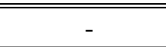 \\
\hline Accuracy (\%) & \multicolumn{6}{|c|}{51.90} \\
\hline
\end{tabular}

Table 6. Confusion matrix of SIMCA based on each species PCA models using Savitzky-Golay $2^{\text {nd }}$ derivative preprocessed spectra.

\begin{tabular}{|c|c|c|c|c|c|c|}
\hline $\begin{array}{l}\text { Actual species } \\
\text { Predicted species }\end{array}$ & Larch & Red pine & Korean pine & Cedar & Cypress & Precision (\%) \\
\hline Larch & 152 & 0 & 0 & 0 & 0 & 100.00 \\
\hline Red pine & 0 & 139 & 1 & 0 & 0 & 99.29 \\
\hline Korean pine & 2 & 0 & 135 & 0 & 0 & 98.54 \\
\hline Cedar & 0 & 0 & 0 & 165 & 0 & 100.00 \\
\hline Cypress & 0 & 0 & 0 & 0 & 139 & 100.00 \\
\hline Unclassified & 37 & 52 & 41 & 33 & 32 & - \\
\hline Multi-classified & 9 & 9 & 23 & 2 & 29 & - \\
\hline Total (ea) & 200 & 200 & 200 & 200 & 200 & - \\
\hline Recall (\%) & 76.00 & 69.50 & 67.50 & 82.50 & 69.50 & - \\
\hline Accuracy (\%) & \multicolumn{6}{|c|}{73.00} \\
\hline
\end{tabular}


cypress after SNV preprocessing was a subset of cedar.

Table 6 lists the species classification result of SIMCA based on Savitzky-Golay $2^{\text {nd }}$ derivative preprocessed spectra. The classification accuracy was $73.00 \%$, and the precisions for each species ranged from 98.54-100.00\%. The recalls for each species was in the range $67.50-82.50 \%$. Accuracy, precision, and recall were dramatically improved after the Savitzky-Golay $2^{\text {nd }}$ derivative preprocessing of raw spectra for every species. The reliability parameters were improved compared to the SNV case. The total amount of unclassified samples was decreased compared to the SIMCA model with raw spectra. The unclassified samples are considered outliers for the model, but this is not a critical problem in classification compared to misclassification. In this context, it was encouraging that the minimum precision was $<98.54 \%$. Although it had relatively low accuracy and recall, only three samples were misclassified as different species in the test set (1,000 samples) by SIMCA based on SavitzkyGolay $2^{\text {nd }}$ derivative preprocessed spectra. As a result, Savitzky-Golay $2^{\text {nd }}$ derivative preprocessing of NIR spectra showed the best reliability in SIMCA classification. The accuracy, minimum precision, and minimum recall of the best model were evaluated as $73.00 \%$, $98.54 \%$, and $67.50 \%$, respectively.

\section{CONCLUSION}

This study examined the NIR spectroscopy and SIMCA for lumber species classification. A SIMCA classification model was developed using the NIR spectra acquired from the lumber surface. The classification reliability indices differed by mathematical preprocessing (raw, SNV, and SavitzkyGolay $2^{\text {nd }}$ derivatives) of the NIR spectra. Among the modeling conditions, Savitzky-Golay $2^{\text {nd }}$ derivatives showed the best classification performance. The best NIR spectra acquired from the lumber could be applied to classify lumber species by the SIMCA classification method, but the accuracy and recall should be improved.

\section{ACKNOWLEDGMENT}

This study was carried out with the support of 'R\&D Program for Forest Science Technology (Project No. 2016009B10-1819-AB01)' provided by Korea Forest Service(Korea Forestry Promotion Institute).

\section{REFERENCES}

Adedipe, O.E., Dawson-Andoh, B., Slahor, J., Osborn, L. 2008. Classification of red oak (Quercus rubra) and white oak (Quercus alba) wood using a near infrared spectrometer and soft independent modelling of class analogies. Journal of Near Infrared Spectroscopy 16(1): 49-57.

Alves, A., Schwanninger, M., Pereira, H., Rodrigues, J. 2006. Calibration of NIR to assess lignin composition (H/G ratio) inmaritime pine wood using analytical pyrolysis as the reference method. Holzforschung 60(1): 29-31.

Barnes, R.J., Dhanoa, M.S., Lister, S.J. 1989. Standard normal variate transformation and de-trending of near-infrared diffuse reflectance spectra. Applied spectroscopy 43(5): 772-777.

Blanco, M. Villarroya, I. 2002. NIR spectroscopy: a rapid-response analytical tool. TrAC Trends in Analytical Chemistry 21(4): 240-250.

Bylesjö, M., Rantalainen, M., Cloarec, O., Nicholson, J.K., Holmes, E., Trygg, J. 2006. OPLS discriminant analysis: combining the strengths of PLS-DA and SIMCA classification. Journal of Chemometrics: A Journal of the Chemometrics Society 20(8-10): 341-351.

Chang, Y.S., Yang, S.Y., Chung, H., Kang, K.Y., Choi, 
Sang-Yun Yang $\cdot$ Yonggun Park $\cdot$ Hyunwoo Chung $\cdot$ Hyunbin Kim $\cdot$ Se-Yeong Park

In-Gyu Choi $\cdot$ Ohkyung Kwon $\cdot$ Hwanmyeong Yeo

J.W., Choi, I.G., Yeo, H. 2015. Development of Moisture Content Prediction Model for Larix kaempferi Sawdust Using Near Infrared Spectroscopy. Journal of the Korean Wood Science and Technology 43(3): 304-310.

Cho, B.K., Lohoumi, S., Choi, C., Yang, S.M., Kang, S.G. 2016. Study on Rapid Measurement of Wood Powder Concentration of Wood-Plastic Composites using FT-NIR and FT-IR Spectroscopy Techniques. Journal of the Korean Wood Science and Technology 44(6): 852-863.

Eom, C.D., Han, Y.J., Chang, Y.S., Park, J.H., Choi, J.W., Choi, I.G., Yeo, H. 2010. Evaluation of surface moisture content of Liriodendron tulipifera wood in the hygroscopic range using NIR spectroscopy. Journal of the Korean Wood Science and Technology 38(6): 526-531.

Esbensen, K.H., Guyot, D., Westad, F., Houmoller, L.P. 2002. Multivariate data analysis-in practice: an introduction to multivariate data analysis and experimental design 5th edition. Camo Process AS.

Fujimoto, T., Tsuchikawa, S. 2010. Identification of dead and sound knots by near infrared spectroscopy. Journal of Near Infrared Spectroscopy 18(6): 473-479.

Hafemann, L.G., Oliveira, L.S., Cavalin, P. 2014. Forest Species Recognition Using Deep Convolutional Neural Networks. $22^{\text {nd }}$ International Conference on Pattern Recognition (ICPR). pp. 1103-1107.

Hermanson, J.C., Wiedenhoeft, A.C. 2011. A brief review of machine vision in the context of automated wood identification systems. IAWA Journal 32(2): 233-250.

Horvath, L., Peszlen, I., Peralta, P., Kelley, S. 2011. Use of transmittance near-infrared spectroscopy to predict the mechanical properties of 1 -and 2-year-old transgenic aspen. Wood Science and Technology 45(2): 303-314.

Hwang, S.W., Lee, W.H., Horikawa, Y., Sugiyama, J.
2015. Chemometrics Approach For Species Identification of Pinus densiflora Sieb. et Zucc. and Pinus densiflora for. erecta Uyeki-Species Classification Using Near-Infrared Spectroscopy in combination with Multivariate Analysis. Journal of the Korean Wood Science and Technology 43(6): 701-713.

Jiang, Z.H., Huang, A.M., Wang, B. 2006. Near infrared spectroscopy of wood sections and rapid density prediction. Spectroscopy and Spectral Analysis 26(6): 1034-1037.

Kwon, O., Lee, H. G., Lee, M. R., Jang, S., Yang, S. Y., Park, S. Y., Choi, I. G, Yeo, H. 2017. Automatic Wood Species Identification of Korean Softwood Based on Convolutional Neural Networks. Journal of the Korean Wood Science and Technology 45(6): 797-808.

Nisgoski, S., de Oliveira, A.A., de Muñiz, G.I.B. 2017. Artificial neural network and SIMCA classification in some wood discrimination based on near-infrared spectra. Wood Science and Technology 51(4): 929-942.

Park, S.Y., Kim, J.C., Kim, J.H., Yang, S.Y., Kwon, O., Yeo, H., Cho, K.C., Choi, I.G. 2017. Possibility of Wood Classification in Korean Softwood Species Using Near-infrared Spectroscopy Based on Their Chemical Compositions. Journal of the Korean Wood Science and Technology 45(2): 202-212.

Pasquini, C. 2003. Near infrared spectroscopy: fundamentals, practical aspects and analytical applications. Journal of the Brazilian Chemical Society 14(2): 198-219.

Porep, J.U., Kammerer, D.R., Carle, R. 2015. On-line application of near infrared (NIR) spectroscopy in food production. Trends in Food Science and Technology 46(2): 211-230.

Russ, A., Fišerová, M., Gigac, J. 2009. Preliminary study of wood species identification by NIR spectroscopy. Wood Research 54(4): 23-32. 
Soft Independent Modeling of Class Analogy for Classifying Lumber Species Using their Near-infrared Spectra

Savitzky, A., Golay, M.J. 1964. Smoothing and differentiation of data by simplified least squares procedures. Analytical chemistry 36(8): 1627-1639.

Schimleck, L.R. Evans, R. 2003. Estimation of air-dry density of increment cores by near infrared spectroscopy. Appita Journal 56(4): 312-317.

Sokolova, M., Lapalme, G. 2009. A systematic analysis of performance measures for classification tasks. Information Processing \& Management 45(4): 427-437.

Thumm, A., Meder, R. 2001. Stiffness prediction of radiata pine clearwood test pieces using near infrared spectroscopy. Journal of Near Infrared Spectroscopy 9(2): 117-122.

Thygesen, L.G., Lundqvist, S.O. 2000. NIR measurement of moisture content in wood under unstable temperature conditions. Part 1. Thermal effects in near infrared spectra of wood. Journal of Near Infrared Spectroscopy 8(3): 183-189.

Üner, B., Karaman, İ., Tanrıverdi, H., Özdemir, D. 2011. Determination of lignin and extractive content of Turkish Pine (Pinus brutia Ten.) trees using near infrared spectroscopy and multivariate calibration. Wood Science and Technology 45(1): 121-134.

Watanabe, A., Morita, S., Ozaki, Y. 2006. A study on water adsorption onto microcrystalline cellulose by near-infrared spectroscopy with two-dimensional correlation spectroscopy and principal component analysis. Applied Spectroscopy 60(9): 1054-1061.

Wold, S. 1976. Pattern recognition by means of disjoint principal components models. Pattern recognition 8(3): 127-139.

Yang, I.C., Tsai, C.Y., Hsieh, K.W., Yang, C.W., Ouyang, F., Lo, Y.M., Chen, S. 2013. Integration of SIMCA and near-infrared spectroscopy for rapid and precise identification of herbal medicines. Journal of Food and Drug Analysis 21(3): 268-278.

Yang, S.Y., Han, Y., Park, J.H., Chung, H., Eom, C.D., Yeo, H. 2015. Moisture content prediction model development for major domestic wood species using near infrared spectroscopy. Journal of the Korean Wood Science and Technology 43(3): 311-319.

Yang, S.Y., Park, Y.G., Chung, H.W., Kim, H.B., Park, S.Y., Choi, I.G., Kwon, O., Cho, K.C., Yeo, H. 2017. Partial Least Squares Analysis on NearInfrared Absorbance Spectra by Air-dried Specific Gravity of Major Domestic Softwood Species. Journal of the Korean Wood Science and Technology 45(4): 399-408.

Zhao, R.J., Huo, X.M., Zhang, L. 2009. Estimation of modulus of elasticity of Eucalyptus pellita wood by near infrared spectroscopy. Spectroscopy and Spectral Analysis 29(9): 2392-2395. 